\title{
The Future Is Now - Will the Real Disease Gene Please Stand Up?
}

\author{
E.R. Martin M.A. Schmidt \\ Center for Genetic Epidemiology and Statistical Genetics, Miami Institute for Human Genomics, \\ University of Miami Miller School of Medicine, Miami, Fla., USA
}

\section{Key Words}

Linkage $\cdot$ Linkage disequilibrium $\cdot$ Family-based tests of association $\cdot$ TDT

\begin{abstract}
The transmission/disequilibrium test (TDT) [Spielman et al.: Am J Hum Genet 1993;52:506-516] has been postulated as the future of gene mapping for complex diseases, provided one is able to genotype a dense enough map of markers across the genome. Risch and Merikangas [Science 1996;273:1516-1517] suggested a million-marker screen in affected sibpair (ASP) families, demonstrating that the TDT is a more powerful test of linkage than traditional linkage tests based on allele-sharing when there is also association between marker and disease alleles. While the future of genotyping has arrived, successes in family-based association studies have been modest. This is often attributed to excessive false positives in candidate gene studies. This problem is only exacerbated by the increasing numbers of whole genome association (WGA) screens. When applied in ASPs, the TDT statistic, which assumes transmissions to siblings are independent, is not expected to have a constant variance in the presence of variable linkage. This results in generally more extreme statistics, hence will further aggravate the problem of having a large number of positive results to sort
\end{abstract}

\section{KARGER \\ Fax +41613061234 \\ E-Mail karger@karger.ch \\ www.karger.com}

(C) 2008 S. Karger AG, Basel

$0001-5652 / 08 / 0662-0127 \$ 23.50 / 0$

Accessible online at: www.karger.com/hhe through. So an important question is how many positive TDT results will show up on a chromosome containing a disease gene due only to linkage, and will they obfuscate the true disease gene location. To answer this question we combined theory and computer simulations. These studies show that in ASPs the normal version of the TDT statistic has a mean of 0 and a variance of 1 in unlinked regions, but has a variance larger than 1 in linked regions. In contrast, the pedigree disequilibrium test (PDT) statistic adjusts for correlation between siblings due to linkage and maintains a constant variance of 1 at unassociated markers irrespective of linkage. The TDT statistic is generally larger than the PDT statistic across linked regions. This is true for unassociated as well as associated markers. To compare the two tests we ranked both statistics at the disease locus, or an associated marker, among statistics at all other markers. The TDT did better job than PDT placing the score of the associated marker near the top. Though, strictly speaking, the TDT in ASPs should be interpreted as a test of linkage and not a test of association, there is a good chance that if a marker stands out, the marker is associated as well as linked. In conclusion, our results suggest that TDT is an effective screening tool for WGA studies, especially in multiplex families.

Copyright $\odot 2008$ S. Karger AG, Basel

Eden R. Martin, PhD

University of Miami Miller School of Medicine

1120 NW 14th St, LC M-860

Miami, FL 33136 (USA)

Tel. +1 305243 2372, Fax +1 305243 1968, E-Mail emartin1@med.miami.edu 


\section{Introduction}

In 1996, Risch and Merikangas [1] advocated the transmission/disequilibrium test (TDT) [2] as the future of gene mapping for complex diseases. They argued this by showing that the TDT is more powerful than non-parametric linkage analysis based on allele sharing in affected sibling pairs (ASPs). Their calculations showed that the required sample size for the TDT to achieve $80 \%$ power can be substantially lower, particularly when the genetic effect is small, as is expected for genes contributing to complex disease. This publication marked a turning point for the family-based genome screens and led to an explosion of TDT applications and methodological extensions.

The major caveat noted in this publication was that the power calculations assumed that the actual risk polymorphism was among the markers screened; thus it had to be assumed that a dense enough array of genetic markers was genotyped to assure a high probability that the true locus, or one in strong linkage disequilibrium (LD), was tested. Risch and Merikangas [1] envisioned a millionmarker screen. At the time, this scope was difficult to imagine, but the charge to solve the 'technological problem' was met with great success. There have been tremendous efforts devoted to discovery and cataloguing of single nucleotide polymorphisms (SNPs) across the human genome. Several high-throughput genotyping platforms have been developed and great effort has been devoted to making them cost-effective. The HapMap project [3] has helped to guide the choice of markers to be included in screens based on patterns of LD. Screens of 550,000 markers are already routine, and it is safe to say that the technological problems of the million-marker screen have been solved.

Why then are there not more successes given the wide application of family-based association studies? This is often attributed to excessive false positives and has even led to requirements by some journals for replication of association studies before publication. This problem will only be exacerbated by the increasing numbers of whole genome association (WGA) screens. This issue was not considered by Risch and Merikangas. In theory, markers unlinked to a disease locus should follow the null distribution and have a false-positive rate at the nominal level, thus the issue is not interesting for unlinked markers. However, when applied in ASPs, the TDT is valid only as a test of linkage; it is not valid as a test for association at linked markers [4]. So an important question is how many positive TDT results will show up by chance on a chromosome containing a disease gene due only to linkage, and will they obfuscate the true disease location.

The pedigree disequilibrium test (PDT) was developed to minimize positive results due to linkage without association when multiplex families are used $[5,6]$. It does this by appropriately adjusting for the correlation of allele transmissions to affected siblings that result due to linkage to a disease locus. By doing so, the PDT provides a test for both association and linkage; thus, the PDT maintains the nominal significance level at linked markers unless they are also associated. This accuracy as a test of association in the presence of linkage makes the PDT applicable for fine-mapping in regions of linkage or candidate gene studies when we want to test specifically for associations.

Paradigms for complex disease gene discovery are changing now with the availability of dense WGA marker panels. We may have no a priori evidence of linkage; so in fact, identifying linkage, irrespective of association, may be informative. In order to maintain the correct significance level as a test of association, the PDT sacrifices power as a test of linkage. The TDT is generally a more powerful test than the PDT for the null hypothesis of no linkage. However, all of the TDT statistics on a linked chromosome will be affected by the linkage, while the PDT statistics all follow the same null distribution unless there is also association. One way to view this is that on a linked chromosome, the PDT and TDT statistics at the actual disease locus, or a marker in LD, are against different backgrounds of chromosome-wide tests. For the PDT, the background maintains the correct nominal significance level, while the TDT statistics should be inflated. This raises the important question of whether the disease locus will stand out better in a dense screen with the TDT or the PDT when we have multiplex families. If so, there would be a reason to reconsider using the original TDT as a screen in WGAs using ASPs instead of requiring a more stringent test that adjusts for linkage to maintain validity as a true test of association.

In this paper, we look specifically at two relevant questions. How many positive TDT results do we expect on a linked chromosome at unassociated markers when ASPs are used? How will these statistics be distributed relative to statistics at the truly associated markers, i.e., will the true disease loci stand out? To answer these questions we derive theoretical approximations for the expected number of positive TDT results at a completely linked but unassociated marker. We then use computer simulations to examine the TDT results as linkage to the disease locus varies. Finally, we use the ordering of TDT statistics 
across a simulated chromosome of 30,000 markers to evaluate how often the test at the true disease locus, or a marker in LD, is in the upper percentiles, or rises to the top. We compare this ordering to that of the PDT to study the impact of adjusting for linkage in multiplex families on the ability to detect the disease locus.

\section{Methods}

\section{The TDT}

In its original form, the TDT is calculated by observing which allele has been transmitted to affected offspring from each heterozygous parent in families with at least one affected offspring. If we assume that a marker has two alleles $M 1$ and $M 2$, then let the number of times that $M 1$ is transmitted be $n_{1}$, then the number of times that it was not transmitted is $n_{2}=h-n_{1}$, where $h$ is the number of heterozygous parents in the sample of families. The TDT statistic is:

$$
T D T^{2}=\frac{\left(n_{1}-n_{2}\right)^{2}}{h}
$$

We write $T D T^{2}$ because it is more convenient work with the normal version of the TDT statistic rather that the original chisquared form. Under the null hypothesis of no linkage, the TDT is asymptotically normal with mean 0 and variance 1 .

When only triad families are used and assuming a multiplicative model (as in Risch and Merikangas) so that parental transmissions are independent, the count, $n_{1}$ is distributed as a binomial random variable:

$$
n_{1} \sim \operatorname{bin}(h, \tau)
$$

where $\tau$ is the probability that allele $M 1$ is transmitted from a heterozygous parent to an affected offspring. The TDT tests the null hypothesis that $\tau=1 / 2$, which gives $E(T D T)=0$ and $\operatorname{Var}(T D T)=$ 1. In triad families, $\tau=1 / 2$ if there is either no linkage or no allelic association (i.e., no LD) between the marker and disease locus; thus the TDT is a valid test of the composite null hypothesis of no linkage or no association in triads.

When families with multiple affected individuals are used, the statistic $n_{1}$ no longer follows a binomial distribution for markers that are linked to the disease locus, even if there is no LD between marker and disease alleles, because transmissions to multiple affected siblings are not independent. Assume that a heterozygous parent has two affected offspring. Let $I_{h}$ be the number of times that the parent transmits $M 1$ to the offspring pair. If there is no LD between the marker and disease locus, the distribution of $I_{h}$ is as follows:

$$
I_{h}=\left\{\begin{array}{lll}
2 & \text { with probability } & 1 / 2 \rho \\
1 & \text { with probability } & 1-\rho \\
0 & \text { with probability } & 1 / 2 \rho
\end{array}\right.
$$

where $\rho$ is the probability that a heterozygous parent transmits the same marker allele to both offspring. The value of $\rho$ depends on the recombination fraction between the marker and disease locus and the disease model, and is equal to $1 / 2$ when there is no link- age. With this parameterization, it is not difficult to show that $E(T D T)=0$ and $\operatorname{Var}(T D T)=2 \rho$, where $1 / 2<\rho<1$. Following this distribution, we can compute the probability that TDT exceeds some critical value $c_{\alpha}$ corresponding to a nominal significance level $\alpha$. Importantly, the variance of the TDT does not depend on allele frequency, thus this distribution holds for any marker not in LD with the disease locus.

\section{The PDT}

The PDT was developed to provide a valid test of both linkage and association in families with multiple affected individuals. The form of the PDT is similar to the TDT, the only difference being the estimate of variance used in the statistic, which allows for correlation between multiple affected individuals. In a sample of $\mathrm{N}$ families, the PDT statistic can be written as

$$
P D T^{2}=\frac{\left(n_{1}-n_{2}\right)^{2}}{\sum_{i=1}^{N} D_{i}^{2}}
$$

where $n_{1}$ and $n_{2}$ are the total number of transmissions from heterozygous parents to affected offspring for alleles $M 1$ and $M 2$, respectively, and $D_{i}$ is the difference between allele counts $n_{1}$ and $n_{2}$ within a single pedigree. If there is no linkage or no association, then $E(P D T)=0$ and $\operatorname{Var}(P D T)=1$. In triad families, the difference between the TDT and PDT is only how families with two heterozygous parents contribute to the variance estimate in the denominator. In multiplex families, the PDT allows for correlations between multiple affecteds by treating entire families as the independent sampling units rather than transmissions from each heterozygous parent.

\section{Computer Simulations}

Computer simulations were run using SIMLA 3.2 [7] and a newly designed, multi-threaded implementation of the TDT algorithm. Focus of our efforts was on two distinct disease models detailed in the Risch and Merikangas [1]. Both models assume a multiplicative mode of inheritance with a disease allele frequency of $P(D)=0.1$. The genotypic relative risks are

$$
\gamma_{D d}=\frac{P(\text { aff } \mid D d)}{P(\text { aff } \mid d d)} \text { and } \gamma_{D D}=\gamma_{D d}^{2}
$$

From the earlier study [1] we used $\gamma_{D d}=2$ and $\gamma_{D d}=4$, and disease prevalence of 0.1 and 0.02 , respectively. We used sample sizes of 264 ASP families for simulations with $\gamma_{D d}=2$ and 48 ASP families for simulations with $\gamma_{D d}=4$. These were the sample sizes computed by Risch and Merikangas that would give approximately $80 \%$ power (with $\alpha=10^{-6}$ ) for the TDT as a test of linkage. In our simulations estimates of power for models with $\gamma_{D d}=2$ and $\gamma_{D d}=4$ were 76.05 and $78.03 \%$, respectively, which are consistent with the previous calculations.

The simulated marker map consists of 30,000 SNPs spaced evenly over 2.0 Morgans. The disease locus was in perfect LD $\left(r^{2}=1\right)$ or partial LD $\left(r^{2}=0.5\right.$ or 0.1$)$ with one SNP having minor allele frequency (MAF) 0.1 or 0.02 . The remaining 29,999 SNPs all had MAF 0.5 and were simulated in linkage equilibrium. We alternately placed the disease-associated SNP at locus 10 (end) or locus 15,000 (mid). For each disease model and disease-associated SNP placement, we simulated replicate datasets consisting of ASP families, each with two genotyped parents with exactly two af- 


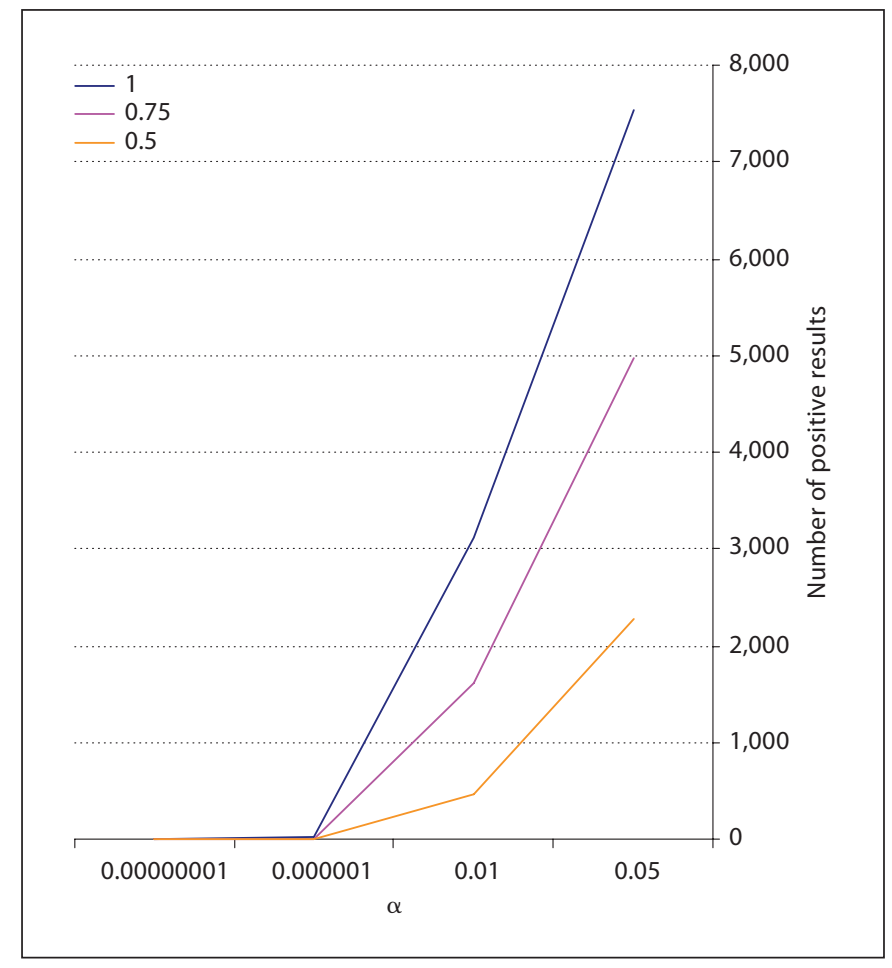

Fig. 1. Expected number of positive results for the TDT in affected sibpairs as a function of $\alpha$ for three values of $\rho$, assuming that tests were conducted at 45,455 biallelic loci.

fected offspring. Estimates of variance and type I error were computed for the TDT over 10,000 replicates. To evaluate the relative ordering of the disease-locus SNP, we used 1,250 replicates.

\section{Results}

How Many Markers Will Be Significant in ASP

Families on a Linked Chromosome?

Figure 1 shows the expected number of positive results (significant at level $\alpha$ ) as a function of $\alpha$ for three values of $\rho$. We assumed that tests were conducted on an average chromosome with 45,455 biallelic markers in linkage equilibrium. We see that the number of positive results increases with increasing significance level $\alpha$, and the increase is much steeper for higher values of $\rho$, representing more allele sharing between siblings. The line at $\rho=0.5$ shows the expected number of positive results when there is no linkage. The other lines show the increase in positive results when there is increased allele-sharing due to linkage. The number of positive results can be substantially larger when there is linkage. For example, if $\rho=0.75$, one would have to sort through more than twice as many positive results than on an unlinked chromosome.

The problem with this graph is that it assumes, for a fixed disease model, that recombination with the disease locus is constant across the markers. To examine the number of positive results allowing recombination to increase with distance from the disease locus, we used computer simulations. Figure 2 shows that for a fixed disease model, the variance of the TDT statistic is larger than 1 , and is largest for marker loci closer to the true disease locus. When the disease locus is in the middle of the chromosome (fig. 2a, c), we see that even at the ends of the simulated chromosome, the variance is larger than it should be if the standard normal distribution of the TDT held. When the disease locus is at one end of the chromosome, the variance does approach 1 at the opposite end (>2.0 M away). The variance is higher for models with higher genetic effects (fig. 2a, b), which was expected since there is a higher degree of allele sharing in these families (larger $\rho$ for a given recombination fraction).

Figure 3 shows the effect of this increased variance on the expected number of positive results. So that we could get accurate estimates with a reasonable number of replicates, we set the nominal significance level at $\alpha=0.05$, rather than the stringent level of $\alpha=10^{-6}$ that Risch and Merikangas used. For the model with $\gamma_{D d}=4$ (fig. 3a), the frequency of positive results ranges from values close to the expected value of 0.05 at the ends of the chromosome to values around 0.08 near the true disease locus. As expected since the variance of the TDT statistic does not depend on allele frequencies, similar results were obtained when we simulated markers with MAF 0.2 (results not shown).

Though not shown here, we verified that the average variance of the PDT was 1 and the false-positive rate was 0.05 across a linked chromosome without a disease-associated marker. We also verified that the frequency of positive results with both TDT and PDT on a chromosome unlinked to a disease gene was consistent with the nominal type I error rate.

Fig. 2. Variance estimates of the TDT at 30,000 marker loci in affected sibpairs from models using $\gamma=2$ and 4 with sample sizes (N) taken from R\&M [1] and the disease locus in the middle or end of the chromosome, $\mathbf{a} \gamma=4, \mathrm{~N}=48$, middle; $\mathbf{b} \gamma=4, \mathrm{~N}=48$, end; $\mathbf{c} \gamma=2, \mathrm{~N}=264$, middle; $\mathbf{d} \gamma=2, \mathrm{~N}=264$, end.

Fig. 3. Frequencies of positive results for the TDT in affected sibpairs from models using $\gamma=2$ and 4 with sample sizes $(\mathrm{N})$ and the disease locus in the middle of the chromosome. a $\gamma=4, \mathrm{~N}=48$; b $\gamma=2, \mathrm{~N}=264$. 

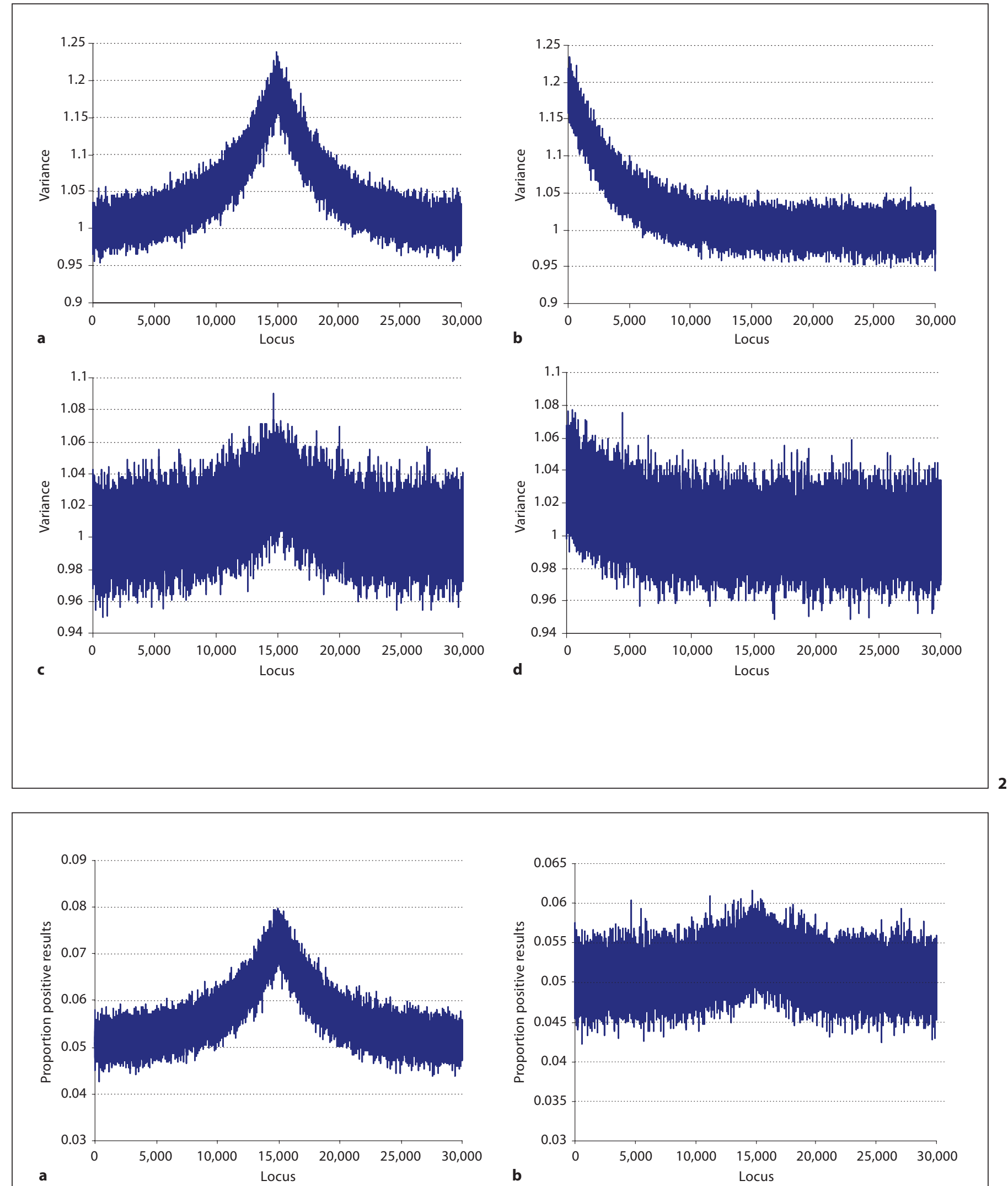


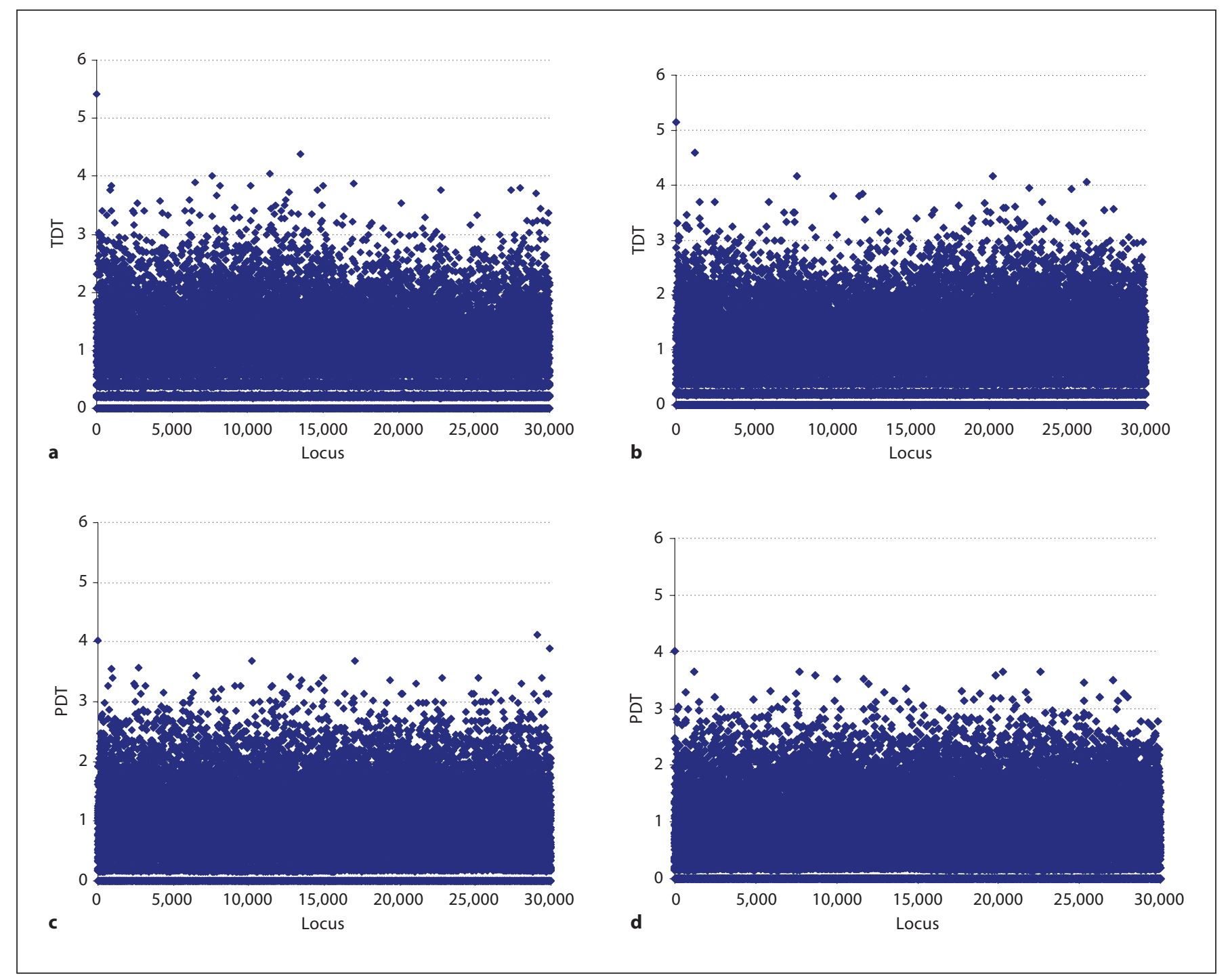

Fig. 4. Two random examples of a SNP screen across a simulated chromosome with TDT (a and b) and PDT (c and $\mathbf{d}$ ). The absolute value of the normal statistics are shown. The disease-associated marker is locus 10 $\left(r^{2}=0.5, \gamma=4\right)$.

How Will Statistics on a Linked Chromosome Be

Distributed Relative to Disease-Associated Loci

(Will the True Positives Stand Out)?

Next, we addressed the question of whether the true positive result at the associated marker will stand out above the background noise across a linked chromosome. Figure 4 shows two examples of replicate screens across our simulated chromosome with $\gamma_{D d}=4$ at a disease locus in LD $\left(r^{2}=0.5\right)$ with marker locus 10 (and all other markers in linkage equilibrium). We see that with TDT (fig. 4a, b), despite the increased variance across the whole chromosome, it is fairly easy to identify the disease-associated marker (locus 10). In both examples the absolute value of the normal TDT statistic at the SNP in LD is over 5.0, while at all other loci the value is no larger than 4.5. We wondered whether the PDT would stand out as well, since it is against a background of tests from the null distribution with a smaller variance of 1 . Figure $4 \mathrm{c}$, d show that the PDT is more ambiguous than TDT. In the first example with the PDT (fig. 4c), the PDT at the diseaseassociated marker is not the largest; instead the largest PDT statistic is generated by a marker at the other end of 
the chromosome. In the second example, the PDT at locus 10 is the largest, but the difference between it and several other loci is relatively small compared to the results from the TDT. These results demonstrate that, the TDT is consistently more significant than the PDT, even at the disease-associated locus leading to a relative loss of power.

To see if these results generalize, we considered the relative ordering, or rank, of the disease-associated marker and the other linked markers over 1,250 replicates. This approach of looking at ranks or precision of gene location is an alternative to statistical power calculations that has been used to evaluate statistical methods in the context of genome scans [8-11]. Specifically, we compared the ordering of TDT and PDT for a disease model with $\gamma_{D d}=4$. When the disease-associated marker was in perfect LD $\left(r^{2}=1\right)$ with the disease allele, it was almost always the most significant with either TDT or PDT. Figure 5 shows where the statistic at the disease-associated marker falls relative to the other markers for different levels of LD. We see that when $r^{2}=0.5$ (fig. 5a), the disease-associated marker is the most significant marker with the TDT for about $2 / 3$ of the replicates, while the disease-associated marker is the most significant with the PDT for only about $1 / 2$ of the replicates. As we relax our criteria, allowing the test to be among the top $x$, we quickly ensure that we include the associated marker in the top percentile. For example, if we take the top $1 \%$ of markers for follow-up, we are virtually assured that we will include the marker in LD for the models that we considered assuming $r^{2}=0.5$. For $r^{2}=0.1$ (fig. $5 b$ ), it is much more difficult to capture the disease-associated marker, but still the TDT does a better job than the PDT.

\section{Discussion}

We set out in this study to revisit the results of Risch and Merikangas [1] in light of the now available dense WGA screens. Their work addressed the power of the TDT in ASPs and was important in promoting the TDT as a family-based test of linkage in the presence of association, but they did not consider the number of positive TDT results expected on a linked chromosome at unassociated markers. Understanding the distribution of TDT scores across chromosomes is key to assessing whether the true disease location can be identified. We have shown here that the TDT has a variance greater than 1 when there is linkage, leading to an increased number of significant results on linked chromosomes. Even small in-

The Future Is Now

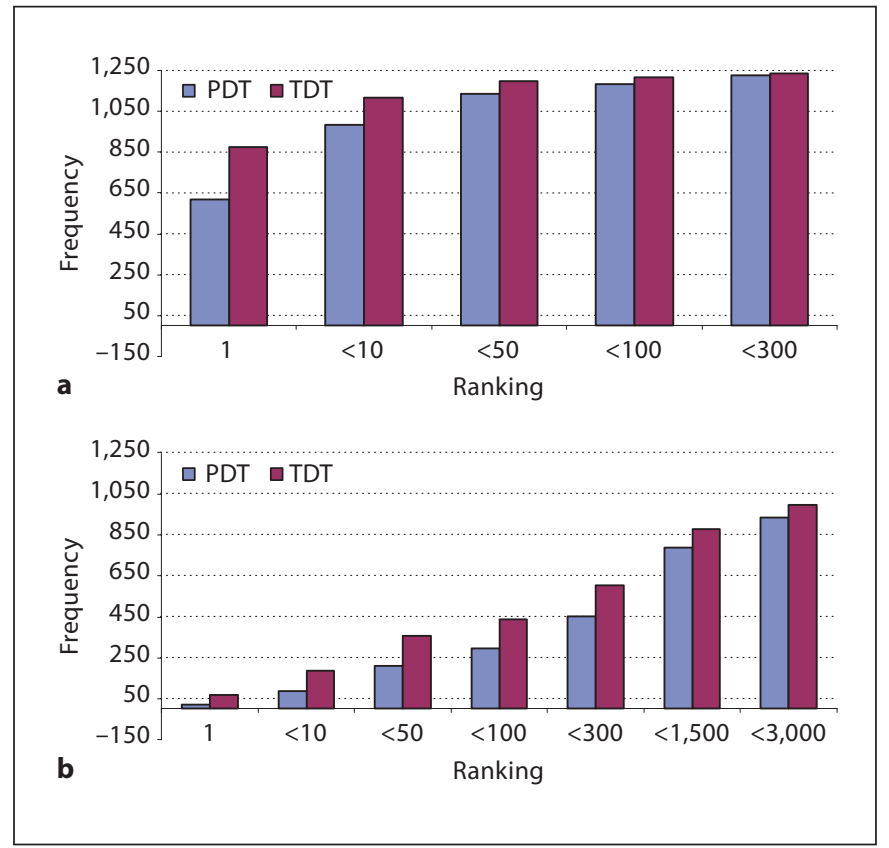

Fig. 5. Histograms showing number of times that the PDT and TDT statistics for a SNP in LD with the disease locus $(\gamma=4)$ are in the top percentiles of statistics for $\mathbf{a} r^{2}=0.5$ and $\mathbf{b} r^{2}=0.1$. The category 1 means that the SNP was the most significant over all SNPs. The category $<\mathrm{x}$ means that the SNP was among the top $\mathrm{x}$ statistics.

flations in significance for the TDT at a single locus can lead to gross inflation across a WGA screen of hundreds of thousands of markers. Compounding this is that, for complex diseases, we expect there to be multiple disease susceptibility loci. Therefore in one sample, there are likely to be several linked chromosomes, and using the TDT in ASPs will result in inflated scores across each of these.

If we had stopped there, we would have concluded that the TDT in multiplex families, as predicted, will produce a lot of background noise to deal with in a WGA; and it may not be as good as methods, like the PDT, that adjust for linkage, eliminating noise due to linkage without association. However, controlling noise is only part of the issue. We also must know how the statistics at a true disease locus, or a marker in $\mathrm{LD}$, are distributed relative to the noise. We really want to know if the true disease loci will stand out. We found that, despite the generally increased number of positive TDT statistics across a linked chromosome, the TDT at the disease-associated marker was often ranked among the top scores, even at a marker with moderate LD. Further, the TDT does a better job 
including the disease-associated marker among the top ranked than the PDT, and this result is robust to the amount of LD between the marker and disease locus. When only a marker with low LD is included, capturing the disease-associated locus among the top ranked is more difficult. One way to increase power at a marker in low LD is to increase the sample size. Notably though, the distribution of the TDT with a fixed level of linkage does not depend on the number of families, $N$, when there is no association. Consequently, increasing sample size will help the disease locus stand out with either TDT or PDT without increasing the noise level.

With the culmination of this study, we are lead to conclude that the TDT is an effective screening tool for WGA studies. It can be applied in multiplex families but will have an excessive number of positive results if an absolute cut-off for significance is used. However, if markers are ranked from most significant to least, the true disease-associated marker is likely to be among the top ranked if sample size is adequate.

We avoided directly discussing statistical power in this article in favor of a discussion of ranks because of the complexities of interpreting a power comparison between TDT and PDT. The statistics test different null hypotheses. In general, the TDT tests the null hypothesis of no linkage, the PDT the null hypothesis of no linkage or no LD. Thus the type I errors of the two tests are not necessarily the same over the same parts of the parameter space. Given this distinction, we did still compute the power of the tests for their respective null hypotheses. For the models with $\gamma_{D d}=2$ and 4 of Risch and Merikangas, which gave estimated powers (at $\alpha=10^{-6}$ ) in our simulated data of 76.05 and $78.03 \%$ for the TDT in ASP families, we found the power of the PDT to be 60.16 and $1.9 \%$ for the two models. We expected the PDT to have lower power than the TDT in ASPs because the PDT must adjust for the positive correlation between allele transmissions to affected siblings which increases the variance estimate used in the statistic. Intuitively, the relative difference between PDT and TDT in the two models can be explained in terms of differences in allele sharing in affected siblings. For large genetic effects, such as the model with $\gamma_{D d}=4$, there is increased allele sharing between affected siblings, leading to an increased correlation in allele transmissions to affected siblings [1] and explaining the substantial power difference between PDT and TDT for this model. As the genetic effect gets smaller and there is less allele sharing in ASPs, the power of PDT approaches that of TDT. For example, for the model with $\gamma_{D d}=1.5$ of Risch and Merikangas [1], we estimated pow- ers of the PDT and TDT as 76.10 and $80.04 \%$. Consequently for genes with small effects, the TDT and PDT are likely to perform similarly; it is genes with moderate or large effects that will benefit most from screening with the TDT.

We did not consider in this article several other interesting questions that arise from the results of this study. One is the impact of LD and genotyping error between markers on results. We would expect LD to give a different distribution of significant results across the genome, but it is unlikely to change the conclusions about the relative ordering of the statistics [8]. It has been shown that genotyping error can result in increased false-positive rates for the TDT [12]. Godon et al. [12] proposed the TDTae as an alternative to the original TDT that adjusts for genotyping error. They found that TDTae has more accurate Type I error and better precision than the original TDT in the presence of genotyping error [11]. Though we did not examine the impact of genotyping error in this article, we expect that TDTae would be a better choice than TDT or PDT for screening when genotyping error is a concern.

Another question is whether applying a correction for multiple tests would change our conclusions about the ranks of statistics. Although adjustments like the Bonferroni correction do not change the ranks of statistics, they do change the distribution of ranks across scans [8]. It would be of interest to study the impact of various multiple testing adjustments on the rank of the TDT. Finally, an important question is how these conclusions might be applied to late-onset diseases for which parental genotype data are often unavailable. In these cases, the relevant comparison is between the Sib-TDT [13] and the PDT in discordant sibships. The PDT applied in discordant sibships adjusts for correlation in genotypes among affected siblings due to linkage, while the Sib-TDT does not. Though we did not consider this here, it is likely that the same conclusions would hold and Sib-TDT would provide a good screening tool for WGAs of late-onset diseases. These remain interesting questions for future exploration.

\section{Acknowledgements}

This work was supported by a grant from the National Institute of Mental Health MH059528. 


\section{References}

1 Risch N, Merikangas K: The future of genetic studies of complex human disorders. Science 1996;273:1516-1517.

2 Spielman RS, McGinnis RE, Ewens WJ: Transmission test for linkage disequilibrium: The insulin gene region and insulin-dependent diabetes mellitus (IDDM). Am J Hum Genet 1993;52:506-516.

3 The international HapMap project. Nature 2003;426:789-796.

4 Martin ER, Kaplan NL, Weir BS: Tests for linkage and association in nuclear families. Am J Hum Genet 1997;61:439-448.

5 Martin ER, Monks SA, Warren LL, Kaplan NL: A test for linkage and association in general pedigrees: The pedigree disequilibrium test. Am J Hum Genet 2000;67:146-154.
6 Martin ER, Bass MP, Kaplan NL: Correcting for a potential bias in the pedigree disequilibrium test. Am J Hum Gen 2001;68:10651067.

7 Schmidt M, Hauser ER, Martin ER, Schmidt $S$ : Extension of the SIMLA package for generating pedigrees with complex inheritance patterns: Environmental covariates, genegene and gene-environment interaction. Stat Appl Genet Mol Biol 2005;4:article 15.

8 Zaykin DV, Zhivotovsky LA: Ranks of genuine associations in whole-genome scans. Genetics 2005; 171:813-823.

9 Satagopan JM, Verbel DA, Venkatraman ES, Offit KE, Begg CB: Two-stage designs for gene-disease association studies. Biometrics 2002;58:163-170

10 Papachristou C, Lin S: A comparison of methods for intermediate fine mapping. Genet Epidemiol 2006;30:677-689.
1 Barral S, Haynes C, Levenstien MA, Gordon D: Precision and type I error rate in the presence of genotype errors and missing parental data: A comparison between the original transmission disequilibrium test (TDT) and TDTae statistics. BMC Genet 2005;6(suppl 1):S150.

12 Gordon D, Haynes C, Johnnidis C, Patel SB, Bowcock AM, Ott J: A transmission disequilibrium test for general pedigrees that is robust to the presence of random genotyping errors and any number of untyped parents. Eur J Hum Genet 2004;12:752-761.

13 Spielman RS, Ewens WJ: A sibship test for linkage in the presence of association: The sib transmission/disequilibrium test. Am J Hum Genet 1998;62:450-458. 